\title{
Patients' internet use in New Zealand for information about medicines: Implications for policy and practice
}

\section{ABSTRACT}

Background: The ubiquitous use of the internet sees patients increasingly look online for information about their medicines.

Objective: This study aimed to understand the use of internet to meet medicine information needs of a sample of New Zealand patients.

Method: Using a descriptive exploratory approach 60 mental health and general medical adult patients at one large urban were interviewed. These semi-structured interviews were audio recorded, transcribed and coded for inductive thematic analysis.

Findings: This study found that the internet is frequently used to meet the medicines information needs of patients. Despite the ease of access to information on the internet patients need guidance to locate credible and trustworthy online resources.

Conclusions: Implications from this study relate to both practice and policy, and include the need for health professionals to have enhanced communication skills as they become information brokers who provide supplementary, reliable sources of patient-centric medicines information. Having a New Zealand specific website that includes an extensive section on medicines is a policy recommendation of this study, as is identifying tools to readily identify patients' needs and preferences for medicines information.

\section{INTRODUCTION}

There has been a marked rise in internet use since 2000 in almost all nations (1). This increase has been identified as essential to a digital economy and a powerful catalyst for 'innovation, economic growth and social wellbeing' (2: p.5), with the potential to benefit not just one nation, but many nations. Harnessing the benefits of the internet for healthcare professionals and patients in terms of facilitating access to health information has been frequently reported (3). The internet provides many with easy, convenient access to health information and the number of health-related websites and patients seeking online health information has grown substantially over the past decade and a half 
(4). Of note, there is currently debate about how equitable this increased access is (5). Of the different types of healthcare information available, information relating to medicines is one of the most important, and a common topic of interest for many patients (3). In this report patient is used to denote an in-patient healthcare consumer, in preference to either client or service-user. Increased access to high quality medicines information written specifically to meet patients' needs has the potential to revolutionise patient self-management and increase adherence to medication regimens. Yet despite the abundance of information available via the internet, there remains a lack of understanding of how New Zealand patients use the internet to access information about medicines. This descriptive, exploratory study aimed to understand the use of internet to meet medicine information needs of a sample of New Zealand patients from one urban hospital. The following report discusses how the internet is currently used by the participating patients to access medicines information, and the potential for the information to influence self-management and medication adherence.

\section{USE OF THE INTERNET}

Studies suggest that patients frequently use the internet to seek information about their medicines, but this is often reported alongside their search for other health information or in specific clinical contexts $(6,7)$. The internet has been used to support medication management in a variety of ways, but the effectiveness of internet-based interventions has been mixed. For example, a 2011 systematic review examining the use of internet-based interventions to improve medication adherence found low to moderate effects on patients' adherence, though the authors note a possible lack of reliability because of the use of self-report measurements (8). A further example is the use of the internet to support the formation of online communities where those with similar interests can network and support each other with their health information needs. However, one small randomised controlled trial in the United Kingdom found that participation in an online community for those with asthma which discussed medications, along with other aspects of health management, did not improve adherence to preventer medication for asthma patients (9). More recently patient portals have been introduced, providing an internet-based option to support patient access to health information, including information about their medicines. One study of 75 patients with diabetes found that whilst 
they were enthusiastic about being able to view their medicines, and order refills of prescriptions, patients wanted additional functionalities of patient portals such as medicines information (10). Car et al. (11) suggest that the internet provides opportunities to support many aspects of a patient's medication management, but that more research is needed, particularly about how medicines information can be personalised. It is hoped that personalising medicines information will support patient engagement and self-management (12).

\section{SELF MANAGEMENT}

The ability for patients to successfully self-manage health conditions is multi-factorial and includes the patient having knowledge of their condition, being able to monitor and manage symptoms and their impact; and adopting lifestyles that promote their health (13). Therefore self-management is dependent on the availability of health and medicines information that meets patient's needs. For more than a decade self-management has been touted as the direction for healthcare to take, particularly to ensure better care for those with long term conditions (14). The aim of selfmanagement is to support the patient to be empowered and become involved in managing their health, including their medicines. The National Institute for Health and Care Excellence (NICE) (15) suggests that this can be led by either the patient or a health professional. Benefits of selfmanagement are wide-ranging - at a national level, by reducing the burden of healthcare costs as patients use the healthcare system less often and more efficiently (16); and at a personal level, by empowering the individual and enabling them to support their own health (17).

The NICE consider patients 'to be active participants in their own healthcare, and to be involved in making decisions about their medicines' (15: p.24). This organisation suggests a number of steps that healthcare professionals should embrace when working with patients, including: 'Offer all people the opportunity to be involved in making decisions about their medicines. Find out what level of involvement in decision-making the person would like and avoid making assumptions about this', then: 'Find out about a person's values and preferences by discussing what is important to them about managing their condition(s) and their medicines. Recognise that the person's values and preferences may be different from those of the health professional and avoid making assumptions about these' (15: p.24-25). In all these steps, information plays an important role in facilitating self- 
management - not only to achieve patient engagement, but specifically in relation to medicines, to improve adherence.

\section{ADHERENCE}

The World Health Organisation defines adherence as: "the extent to which a person's behaviour taking medication, following a diet, and/or executing lifestyle changes—corresponds with agreed recommendations from a health care provider" (18). Much is written about the need to increase medication adherence. In 2014, a Cochrane review of interventions to improve the safe and effective use of medicines was conducted. This review found mixed results, after having synthesised the evidence, irrespective of disease, medication, population, or setting, on the effectiveness of interventions to improve patients' medication use (19). Of note, the authors report that the 75 systematic reviews included had varied research aims including support for behaviour change, risk minimisation and skills acquisition, but none aimed to promote patient participation in medicationrelated activities, even though medication adherence was the most frequently-reported outcome. The review also found that strategies providing information or education as a single intervention appear ineffective to improve medication adherence, but are effective for improving knowledge, which is key for supporting patient choice (19). In contrast, Kardas, Lewek, and Matyjaszczyk's review of systematic reviews of the determinants of medication adherence (20), found having knowledge and information that patients found appropriate, applicable and easily accessible was important for improving adherence. Although these reviews show contrasting findings of the relationship between medicine information and adherence, they both demonstrate that information plays a key role in facilitating patient choice - an essential aspect of adherence. Lamiraud and Geoffard (21) provide a thought provoking consideration regarding adherence by suggesting that medication non-adherence is a choice, meaning that healthcare professionals need to ensure a patient is fully informed to make that decision, and then respect their decision. Similarly, the NICE Guideline on Medicines Optimisation emphasise the responsibility of healthcare professionals to make decisions appropriate to the circumstances of the individual patient, in consultation with the patient and/or their carer or guardian (15), thereby highlighting the importance of recognising and addressing the needs of the individual, whatever they may be. 
The literature confirms that many patients wish to be active participants in their own healthcare and medication management, and that increased access to reliable information is an essential aspect of facilitating this active participation (15). The internet lends itself to being an important information source which can increase ease of access to health information. This study aims to understand the use of the internet to meet medicines information needs of a sample of New Zealand healthcare patients. It is anticipated that the outcomes of this study could be used to guide policy and inform the development of online services to address any medicines information needs identified.

\section{NEW ZEALAND AS CONTEXT FOR THIS STUDY}

New Zealand was the setting for this study. In line with international trends and current evidence, (14, $15,17,22)$ New Zealand's healthcare system is increasingly focusing on patients and their ability to self-manage their own health conditions and medicines. At a national level this is articulated within the 2016 New Zealand Health Strategy, which acknowledges 'people power' as one of its key strategies that aims to empower people to be more involved in their health (23). Healthcare in New Zealand is managed by the Ministry of Health with funding, gained primarily from taxation, devolved to District Health Boards (DHBs) (23). The DHBs coordinate primary, secondary and tertiary healthcare within their region and these are free to citizens.

New Zealand has a population of approximately 4.8 million of which, approximately $88.6 \%$ are internet users (24). In 2014, a New Zealand study surveyed 1783 patients in a primary healthcare setting to gain an understanding of their health information requirements, and found the internet was the third most preferred source of health information, after their doctor/health centre and nurse (7). An earlier New Zealand study, using a brief phone and mall intercept survey, explored patients' use of the internet (25). This study reported that the three most common reasons for searching the internet were for general health or nutrition information ( $45 \%$ of respondents), information about a specific illness (42\%), and for medicines information (40\%). However, neither studies specifically explored patients' use of the internet to meet their medicines information needs. 


\section{METHODS}

This study explored patients' use of the internet to access medicines information as part of a larger study, which investigated patients' needs and preferences for information about their medicines using a descriptive, exploratory approach (26). Data collection took place between September 2016 and March 2017 using semi-structured, face-to-face interviews and a focus group with some patients. Participants were patients either admitted to adult mental health services (acute wards or communitybased rehabilitation mental health centre), or under the care of general medical services of a large urban hospital in New Zealand. Inclusion criteria were those aged 18 years or older, able to speak and understand English, taking at least one regular medication and consenting to participate in the study (Table 1). Potential participants were identified by an independent clinical pharmacist and then referred to the research team. Purposive sampling was used to ensure that patients were recruited from the main ethnic groups residing within the hospital catchment area. Participants were given the opportunity to have a support person present, including kaiatawhai (Māori cultural support workers) for Māori participants; however the opinions of support people were not included in data collection.

The interviews and the focus group were conducted using a semi-structured interview discussion guide (Table 2), where patients were asked to share their needs and preferences for information about their medicines, including their opinions of using the internet to access information for their medicines. Any clinical questions, or specific medicines information needs or concerns were passed on to the ward nurse or pharmacist for follow-up. The focus group and all interviews were audio recorded, except where participants did not give consent, in which case written notes were used. Audio recordings were independently transcribed verbatim and verified by the research team. Following coding of the text using NVivo software (version 11) (27), an inductive thematic analysis (28) of the transcripts was used to extract, describe, and explain the use of the internet to meet medicine information needs of participants. According to Braun and Clark (2006), the use of an inductive approach means the themes are strongly linked to the data, rather than extracting data that fits the beliefs of the researchers. Ethical approval was gained from the New Zealand Health and Disability Ethics Committee and institutional approval from the hospital was also obtained (Ref: 16/NTA/49). 


\section{FINDINGS}

In total 60 patients participated in the study: 30 in-patients under the care of general medical services and 30 mental health service users, 26 of who had been admitted to an acute care setting. The four other mental health service users were in the care of a community-based rehabilitation mental health centre and participated in a focus group interview held there. The interviews lasted between 10 and 45 minutes and all were voice recorded, except for one participant who requested that their interview was not and written notes were taken instead.

The demographic data of participants are shown in Table 3. The mean age of participants was 46 years $(S D=20)$, though participants under the care of general medical services tended to be older (mean=56, SD=20) than those from mental health services (mean $=36, S D=14)$. Of the 60 participants, just under half identified as being New Zealand European $(n=26,43 \%)$, with $30 \%$ identifying as Māori, 17\% a Pacific Island ethnicity, 7\% Asian and 3\% other ethnicities (Table 3). Participants cited Google $\AA$, Wikipedia $\AA$ and WebMD $\AA$ as websites that they commonly used to access medicines information. Social media was rarely used, with the exception of some participants being part of online chat groups for specific mental health conditions. However these social media groups were primarily used for support rather than for access to medicines information

Three key themes were identified: Internet as an enabler, benefits of internet use and barriers to using the internet for medicines information. These are described below using illustrative quotes from participants.

\section{Internet as an enabler}

The internet was used by most participants to search for particular information on their medicines. Medicine information searched for on the internet included the mechanism of action of the medication, side effects, formulation, safety, indications, and efficacy. Participants described various reasons for using the internet to search for medicines information. These included using the internet to bridge unmet medicines information needs due to a lack of information from healthcare providers, or not 
understanding the information that they were provided: "Don't always understand it, so, hence the reason you Google". Participants also turned to the internet to look up medical terminology, to clarify their diagnosis, or to look for alternative options to their medication. For example, one participant commented that the internet "allowed you [the participant] to self-diagnose".

\section{Benefits of internet use}

Several benefits were described by participants. The internet allows participants to access information in a way that is easier and more convenient for them, with a participant commenting that it was "much easier to access something [information via the internet] from home". Others sometimes used the internet as they found it easier to get information online than getting an appointment with a healthcare professional. Furthermore, some participants described the advantages of using the internet to access information in their preferred language, and an additional benefit was finding information that was "more generic and easier to understand".

\section{Barriers to using the internet for medicines information}

Despite the benefits of internet use overall, participants preferred interaction with a healthcare professional over using the internet for medicines information. There was a general concern among participants that the internet lacked reliability and was therefore not a trustworthy source of information. Participants discussed "Google doctoring", stating they looked up their medications and symptoms but that "I [the participant] don't always believe everything I read on the internet". Even though the validity of the information was doubted, participants still reported using the internet to get medicines information because "I don't have any other ways to get my information". Participants suggested that use of the internet could be improved by providing a trustworthy site such as one affiliated with a hospital or government department: "If there was a guaranteed [website], maybe a site that was actually connected to the hospital, that would make me feel a little bit better". Others thought that the internet gave access to excessive information which may contribute to fears or anxiety, such that one participant commented: "I'm too scared to look up the side effects", after they had been surprised by the number and detail of possible side effects of their medications. Some participants recognised that those with access to online resources would find it easier to get the medicines 
information they wanted, and that those with limited access would face a barrier to meeting their healthcare and information needs.

\section{DISCUSSION}

Overall, this study found that while the internet can be an easily accessible, convenient source of medicines information, participants did not want online information to replace interactions with health professionals. This is in line with the findings of Honey et al (7) who found patients' preferred source of health information was a health professional; and also the NICE (2015) guidelines that suggest that consultation with a health professional should be the first option, but that the use of technology, such as internet-based medicines information, might follow.

The internet has an important role to play in the efficient and equitable provision of health-related information (25) however, participants in this study recognised the potential for inequities by acknowledging the possibility that those without access to online resources could face a barrier to accessing information. This has been called the 'digital divide' describing the disparities between those with and without internet access (29). However, this has now been extended from the original 'digital divide' that just related to access to the internet, to overall use of the internet, which raises questions about health literacy (29) and technology literacy (30).

Participants in this study did not consider the internet to be a completely trustworthy source of medicines information. The reasons for this were not clearly elucidated, though the large amount of information, and questionable credibility of some websites, may be contributing factors (31). Health literacy was not explicitly explored in this study, but could be an issue influencing trust and engagement with the internet; a lack of health literacy making the volume and variable quality of internet-based medicines information harder to process. Understanding patient's health literacy skills in relation to their medicines information needs and use of the internet to locate, understand and use credible information about medicines is an area for further study. However, irrespective of health literacy, health professionals have an emerging role in terms of being an information broker or mediator, helping patients to access, understand and source appropriate online information (32). 
This study highlights the importance of trustworthiness and the need for trustworthy websites for providing accessible, accurate medicines information tailored for patients. Using Google® or other search engines to locate medicine-related resources and generic websites such as Wikipedia ${ }^{\circledR}$ can be useful, but the information found, including information from overseas health-related sources such as WebMD®, may not be relevant for New Zealand. For example, access to medicines in New Zealand is controlled centrally by government agencies: Medsafe are responsible for the regulation of medicines and PHARMAC decides which medicines are publicly funded. An information source describing medicines or brands of a medicine not approved for use, or funded in New Zealand could lead to patient dissatisfaction, confusion, or anxiety. Having a central information resource, which provides New Zealand-specific information could potentially be helpful and is an area for further investigation. However, New Zealand does have government subsidised websites where credible medicines information can be found, for example Health Navigator (www.healthnavigator.org.nz) provides a range of health-related information, including some useful information on medicines. New Zealand also has the freely accessible Medsafe website (www.medsafe.govt.nz) which has some information about medicines targeted at patients and the New Zealand Formularies for medicines information (www.nzfc.org). However, these sites are more suited to health professionals and this can sometimes make specific information difficult to locate and/or understand for patients. The findings of this study support the further development and increased promotion of the Health Navigator website to patients. One suggestion of a website to model for the breadth of coverage is NHS Choices (www.nhs.uk), a government funded website in the United Kingdom, which offers information from a central source, including some medicines information, with language translation options. This notion is further supported by the results of a New Zealand based study that highlighted the need for credible resources about medicines on the internet (25). Scott, Scott, \& Auld (2005) stated that because of the risks of patients accessing unreliable information 'a valuable public health policy initiative would be to provide an improved New Zealand health information website containing information on how to evaluate data sourced from the world-wide-web and links to a range of useful and trustworthy health information sites' (25). 
Findings from this study also highlight implications for policy and practice. The professional bodies for healthcare professionals, and the organisations employing health professionals should be supported to provide ongoing education in communication skills as part of all health professional development. This would support both health and non-healthcare staff to communicate effective health information across multiple modes of delivery, and be able to work in collaborative partnerships with patients. In addition, this training should also be provided within undergraduate healthcare professional curricula. Health professionals will need to develop extended communication skills and become effective information brokers to maximise the benefit of face-to-face and online interactions with patients, their family, and/or caregivers. As patients increasingly turn to the internet for medicines information, at a national level, there is need for continued support and promotion of a website that contains New Zealand-specific medicines information in an easy to navigate format. Increasing the awareness of healthcare providers to such a site would also be beneficial for their increasingly important patient information broker or mediator role.

\section{Limitations and areas for further research}

This was an exploratory study, though by using individual face-to-face and focus group interviews, indepth data was collected. The context for this study was one urban hospital and participants were not representative of the New Zealand population. Two sub groups of patients were included in this study: those admitted to adult mental health services (acute wards or community-based rehabilitation mental health centre), or under the care of general medical services. We were not seeking to compare the two groups, rather to understand their use of the internet to meet their medicine information needs. While the patient sub-groups were different demographically the thematic analysis was carried out across all participants. Despite the diversity of participants in this study, and the use of purposive sampling to recruit participants, participant demographics were not similar to the overall New Zealand population, apart from gender. At the latest census (2013), the New Zealand population consisted of $51 \%$ females, and this study had $50 \%$ females (33). The study population was not representative in terms of ethnicity as it had a smaller (43\%) proportion of people who identified as NZ European, compared to the general population (74\%); Pacific Island (17\% vs. general population $7 \%$ ); or Asian (7\% vs. general population 12\%); and a higher proportion of participants identified as being Māori (30\% vs. general population 15\%) (33). Although the demographics of our participants were not 
representative of New Zealand, they closely matched the ethnicity and gender of the study hospital catchment area population. Therefore repeating this study in other areas and other settings, including rural settings of New Zealand and with a larger sample may be warranted. There is also a place for a larger study in order to confirm these findings and to clarify the needs of different ages, ethnicities and varying educational levels.

The themes generated from this study have been used to inform the development of a questionnaire which we plan to administer to larger numbers to confirm the medicines information needs found, but in the meantime, we can take reassurance from the fact that the identified themes are in line with most of the international findings. In the future, and aligned with the NICE Guidelines (15), there is an opportunity for identifying and/or developing validated tools that can be widely used in a range of clinical settings to quickly assess patient's preferences for receiving medicines information, whether mediated by technology or delivered face-to-face.

\section{CONCLUSION}

This study demonstrates that the internet is frequently used to meet the medicines information needs of mental health and general medical adult patients at one large urban New Zealand hospital. Despite the benefits of easy access to plentiful information on the internet, participants would like guidance to locate trustworthy online resources if they require further information about their medicines. Ideally patients should still have interaction with a healthcare professional. This has implications for enhanced communication skills for health professionals, as they become information brokers, who can discuss and provide supplementary, reliable sources of patient-centric medicines information. Having a heavily promoted New Zealand specific website with a wide scope, including an extensive section on medicines in a format aimed at meeting the needs of patients, is a recommendation of this study, as is identifying tools to readily identify patients' needs and preferences for medicines information.

\section{ACKNOWLEDGEMENTS}


We are grateful to the participants for their time and also the ward pharmacists in the mental health team and general medical team who helped with identifying and recruiting participants and the ward staff who supported this study.

Funding: A small project grant was received from Auckland DHB Charitable Trust

Competing interests: None declared

\section{REFERENCES}

1. Internet World Stats. Internet usage statistics: The internet big picture 2017 [cited 2017 November 5]. Available from: http://www.internetworldstats.com/stats.htm

2. Organisation for Economic Co-operation and Development (OECD). Economic and social benefits of internet openness. Committee on Digital Economy Policy (CDEP), OECD; 2016.

3. Fox S, Duggan M. Pew Research Center's Internet \& American Life Project: Health online 2013. Washington D.C., USA: Pew Research Center's Internet \& American Life Project; 2013.

4. Amante DJ, Hogan TP, Pagoto SL, English TM, Lapane KL. Access to care and use of the internet to search for health information: Results from the US National Health Interview Survey. Journal of Medical Internet Research. 2015;17(4):e106.

5. Jacobs W, Amuta AO, Jeon KC. Health information seeking in the digital age: An analysis of health information seeking behavior among US adults. Media \& Communication Studies. 2017;3(1):1302785.

6. Bulut H, Tannkulu G, Dal U, Kapucu S. How much do E.D. patients know about medication prescribed for them on discharge? A pilot study in Turkey. Journal of Emergency Nursing. 2013;39(3):e27-e32.

7. Honey MLL, Roy DE, Bycroft J, Boyd M, Raphael D. Promoting the meaningful use of health information for New Zealand consumers. Studies in Health Technology and Informatics. 2014;201:117. 
8. Linn AJ, Vervloet M, van Dijk L, Smit EG, Van Weert JC. Effects of eHealth interventions on medication adherence: A systematic review of the literature. Journal of Medical Internet Research. 2011;13(4):e103.

9. Koufopoulos JT, Conner MT, Gardner PH, Kellar I. A web-based and mobile health social support intervention to promote adherence to inhaled asthma medications: Randomized controlled trial. Journal of Medical Internet Research. 2016;18(6):e122.

10. Osborn CY, Satterwhite Mayberry L, Wallston KA, Johnson KB, lasy TA. Understanding patient portal use: Implications for medication management. Journal of Medical Internet Research. 2013;15(7):e133.

11. Car J, Tan WS, Huang Z, Sloot P, Franklin BD. eHealth in the future of medications management: Personalisation, monitoring and adherence. BMC Medicine. 2017;15(73):1-9.

12. Horne R. The human dimension: Putting the person into personalised medicine. The New Bioethics. 2017;23(1):38-48.

13. Flinders Human Behaviour \& Health Research Unit. Educating future health care professionals to support people with chronic conditions to live better and live longer. Adelaide, Australia: Flinders University; 2007.

14. World Health Organisation. Innovative care for chronic conditions: Building blocks for action: Global report. Geneva: World Health Organisation; 2002.

15. National Institute for Health and Clinical Excellence. Medicines optimisation: The safe and effective use of medicines to enable the best possible outcomes. London, United Kingdom: NICE; 2015.

16. NHS England. Involving people in their own health and care: Statutory guidance for clinical commissioning groups and NHS England. Leeds, England; 2017.

17. Mental Health Foundation. Surviving or thriving? The state of the UK's mental health. London, United Kingdom; 2017. 
18. Sabaté E, editor. Adherence to long-term therapies: Evidence for action. Geneva, Switzerland: World Health Organization; 2003.

19. Ryan R, Santesso N, Lowe D, Hill S, Grimshaw J, Prictor M, et al. Interventions to improve safe and effective medicines use by consumers: An overview of systematic reviews. The Cochrane Database of Systematic Reviews. 2014;29(4):CD007768.

20. Kardas P, Lewek P, Matyjaszczyk M. Determinants of patient adherence: A review of systematic reviews. Frontiers in Pharmacology. 2013;4(91):1-16.

21. Lamiraud K, Geoffard P-Y. Therapeutic non-adherence: A rational behaviour revealing patient preferences? Health Economics. 2007;16:1185-204.

22. NHS Digital. Patient experience 2017. Available from: http://content.digital.nhs.uk/patientexperience

23. Ministry of Health. New Zealand Health Strategy: Future direction Wellington, New Zealand: Ministry of Health; 2016. Available from:

http://www.health.govt.nz/system/files/documents/publications/new-zealand-health-strategyfuturedirection-2016-apr16.pdf

24. Internet World Stats. Internet usage and population in Oceania 2017 [cited 2017 August 10]. Available from: http://www.internetworldstats.com/stats6.htm\#oceania

25. Scott WG, Scott HM, Auld TS. Consumer access to health information on the internet: Health policy implications. Australia and New Zealand Health Policy [Internet]. 2005;2(13):1-9.

26. Hennink M, Bailey A, Hutter I. Qualitative research methods. London, England: Sage; 2011.

27. QSR International. What is NVIVO? 2017. Available from: www.qsrinternational.com/what-isnvivo

28. Braun V, Clarke V. Using thematic analysis in psychology. Qualitative Research in Psychology. 2006;3(2):77-101. 
29. Büchi M, Just N, Latzer M. Modeling the second-level digital divide: A five-country study of social differences in internet use. New Media \& Society,. 2016;18(11):2703-22.

30. Blake C. Defining technology literacy: Skills students need for personal and professional success Seward, NE: Concordia University; 2015 [cited 2017 November 7]. Available from: https://online.cune.edu/defining-technology-literacy/

31. Sbaffi L, Rowley J. Trust and credibility in web-based health information: A review and agenda for future research. Journal of Medical Internet Research. 2017;19(6):e218.

32. Christozov D, Toleva-Stoimenova S. The role of information brokers in knowledge management. Online Journal of Applied Knowledge Management. 2014;2(2):109-19.

33. Statistics New Zealand. 2013 Census: Quickstats about national highlights. Wellington, New Zealand; 2013. 